Agrotrópica 33(3): 205 -214. 2021.

Centro de Pesquisas do Cacau, Ilhéus, Bahia, Brasil

\title{
CONSERVAÇÃO PÓS-COLHEITA DE FRUTOS DE MARACUJÁ-ALHO BRS VITA FRUIT PRODUZIDOS EM SISTEMA CONVENCIONAL E ORGÂNICO
}

\author{
Maria Madalena Rinaldi', Ana Maria Costaㄹ, Samara Figueiredo de Oliveira Assis Braz ${ }^{2}$, \\ Débora Figueiredo de Oliveira da Silva Assis ${ }^{2}$
}

\begin{abstract}
'Empresa Brasileira de Pesquisa Agropecuária (Embrapa)/ Embrapa Cerrados (CPAC) BR 020, km 18, Rodovia BrasíliaFortaleza, Caixa Postal: 08223, 73310-970, Planaltina, DF, Brasil.madalena.rinaldi@embrapa.br, ana-costa@embrapa.br ${ }^{2}$ Faculdade UnB Planaltina - FUP - Vila NS de Fátima - Vila Nossa Sra. de Fátima, 73345-010, Planaltina - Brasília - DF, Brasil.figueiredosama@gmail.com,dfoassis@gmail.com
\end{abstract}

\begin{abstract}
A Passiflora tenuifila tem sido estudada como alternativa para a indústria farmacêutica devido a sua característica de possuir propriedades para controle de tremores causados pela doença de Parkinson. Devido ao aroma característico de seus frutos é conhecida como maracujá-alho sendo uma espécie silvestre, não havendo informações sobre a conservação pós-colheita. Assim, avaliou-se a vida útil de frutos de P. tenuifila produzidos em sistema convencional e orgânico acondicionados em embalagem PEBD com $30 \mu \mathrm{m}$ de espessura e armazenados sob condição ambiente $\left(23^{\circ} \mathrm{C}\right.$ e $77 \%$ de umidade relativa) e refrigerada $\left(10^{\circ} \mathrm{C}\right.$ e $90 \%$ de umidade relativa) por 10 dias. Analisou-se a perda de massa fresca, concentração de $\mathrm{O}_{2}$ e $\mathrm{CO}_{2}$ no interior das embalagens, luminosidade, $\mathrm{a}^{*}, \mathrm{~b}^{*}$, incremento no escurecimento, croma, ângulo hue, textura, $\mathrm{pH}$, acidez titulável, sólidos solúveis e Ratio. Frutos de P. tenuifila devem ser armazenados em condição refrigerada com temperatura de $10{ }^{\circ} \mathrm{C}$ e $90 \%$ de umidade relativa. Não há diferença significativa na vida útil de frutos de $P$. tenuifila produzidos em sistema convencional e orgânico. Frutos de $P$. tenuifila acondicionados em embalagem de PEAD com $30 \mu \mathrm{m}$ de espessura apresentam maior vida útil por meio da redução da perda de massa fresca, manutenção da cor, textura e características físico-químicas.
\end{abstract}

Palavras-chave: Passiflora tenuifila, vida útil, embalagens, armazenamento, sistema de produção.

\footnotetext{
Postharvest conservation of passion fruit BRS Vita Fruit produced in conventional and organic systems. Passiflora tenuifila has been studied as an alternative for the pharmaceutical industry due to its characteristic of having properties to control tremors caused by Parkinson's disease. Due to the characteristic aroma of its fruits, it is known as passion fruit, being a wild species, with no information on post-harvest conservation. Thus, the shelf life of $P$. tenuifila fruits produced in a conventional and organic system, packed in $30 \mu \mathrm{m}$ thick LDPE packaging and stored under ambient conditions $\left(23^{\circ} \mathrm{C}\right.$ and $77 \%$ relative humidity) and refrigerated $\left(10{ }^{\circ} \mathrm{C}\right)$ was evaluated and $90 \%$ relative humidity) for 10 days. The loss of fresh mass, concentration of $\mathrm{O}_{2}$ and $\mathrm{CO}_{2}$ inside the packages, luminosity, a*, b*, increase in browning, chroma, hue angle, texture, $\mathrm{pH}$, titratable acidity, soluble solids and Ratio were analyzed. Fruits of $P$. tenuifila must be stored in a refrigerated condition at a temperature of $10{ }^{\circ} \mathrm{C}$ and $90 \%$ relative humidity. There is no significant difference in the shelf life of $P$. tenuifila fruits produced in conventional and organic systems. Fruits of $P$. tenuifila packaged in HDPE packaging $30 \mu \mathrm{m}$ thick have a longer shelf life by reducing the loss of fresh mass, maintaining color, texture and physicochemical characteristics.
}

Key words: Passiflora tenuifila, shelf life, packaging, storage, production system. 


\section{Introdução}

O Brasil é o maior produtor e exportador mundial de maracujá (Faleiro et al., 2017) sendo que aproximadamente $95 \%$ dos pomares brasileiros são cultivados com o maracujá amarelo ou azedo (Passiflora edulis Sims) onde o gênero Passiflora é composto por mais de 500 espécies (Faleiro et al., 2020), sendo que muitas delas apresentam potencial de uso alimentar, ornamental e funcional medicinal (Faleiro et al., 2020). Várias espécies do gênero Passiflora são cultivadas comercialmente no mundo, sendo que a espécie Passiflora tenuifila Killip está entre as espécies com potencial de uso funcional-medicinal (Carlosama et al., 2020). Esta espécie tem sido trabalhada no programa de melhoramento genético dos maracujás realizados pela Embrapa Cerrados e parceiros visando o aumento da produtividade e da qualidade física e química de frutos, considerando suas propriedades medicinais-funcionais (Faleiro et al., 2018).

Já foi desenvolvida a BRS Vita Fruit que é uma cultivar de maracujazeiro medicinal da espécie $P$. tenuifila com propósitos de processamento de produtos funcionais-medicinais sendo uma alternativa para $o$ mercado de frutas especiais (Holanda et al., 2020; Pereira, Silveira e Costa, 2017; Faleiro et al., 2018). Esta espécie é popularmente conhecida como maracujá alho devido ao aroma característico de seus frutos. Além das suas propriedades medicinaisfuncionais a espécie possui alta produtividade e qualidade física e química de frutos. Devido às características benéficas, os frutos de $P$. tenuifila podem ser utilizados como matéria-prima na indústria alimentícia, química e farmacêutica (Holanda et al., 2020; Holanda et al., 2019; Silveira et al., 2019).

A aparência externa é um dos principais critérios considerados quando o fruto é destinado ao mercado "in natura", sendo que no maracujá ocorre a perda de massa e o consequente murchamento conferindo aspecto enrugado ao fruto logo após a colheita (Rinaldi, Dianese e Costa, 2021). Outros fatores que atuam no processo de redução da vida útil pós-colheita desses frutos é a susceptibilidade à podridão e a fermentação da polpa (Tavares et al., 2003). Em condições normais, a temperatura e umidade relativa ambiente, Rinaldi et al (2019a) obtiveram vida útil de três a sete dias para frutos de P. edulis ou P. alata. Em frutos de Passiflora setacea o período de vida útil pós-colheita pode ser ainda menor (Rinaldi et al., 2017a e 2017b). Já para frutos de Passiflora cincinnata cv. BRS Sertão Forte a vida útil pós-colheita é significativamente superior (Rinaldi, Dianese e Costa, 2021). Considerando a importância do gênero Passiflora para o nosso país, boas práticas de pós-colheita vêm sendo estudadas e recomendadas (Rinaldi et al., 2019b).

De acordo com a Instrução Normativa Conjunta 2/ 2018 (ANVISA, 2018) os produtores de maracujá devem priorizar a qualidade dos frutos a fim de atender as exigências do mercado. $\mathrm{O}$ uso de tecnologias objetivando a melhoria de tratamentos pós-colheita, armazenamento refrigerado, dentre outros, devem ser priorizados (Zacharias, Faleiro e Almeida, 2020). Essas tecnologias também irão auxiliar na redução de perdas pós-colheita destes frutos, que ainda é significativa a nível de campo, armazenamento e comercialização.

Os atributos de qualidade à comercialização "in natura" e utilização da polpa na elaboração de produtos industrializados devem ser mantidos, por meio de técnicas adequadas de conservação pós-colheita, incluindo à longevidade e condições de armazenamento de frutos de P. tenuifila (Holanda et al., 2019). A utilização de embalagens no acondicionamento de frutos de Passifloras como alternativa para o aumento da vida útil também tem sido estudada e recomendada (Rinaldi et al., 2019a e 2019b; Rinaldi et al., 2017b). A embalagem, quando adequada, auxilia na redução de perda de massa fresca, manutenção da cor, textura e características físico-químicas dos frutos.

Estudos de conservação pós-colheita com a recomendação do manuseio e formas adequadas de armazenamento para frutos de $P$. tenuifila produzidos em sistema convencional e orgânico ainda não foram publicados. Assim, no presente trabalho, objetivou-se avaliar a conservação pós-colheita de frutos de $P$. tenuifila cultivar BRS Vita Fruit produzidos em sistema convencional e orgânico acondicionados em embalagem PEAD e armazenado em diferentes temperaturas.

\section{Material e Métodos}

Utilizaram-se frutos da espécie Passiflora tenuifila cv. BRS VF (nome comercial BRS Vita Fruit) oriundos do campo experimental da Embrapa Cerrados 
(15³6"13.02"S; 4743"17.34"O) a uma altitude de aproximadamente $1050 \mathrm{~m}$, em Planaltina, Distrito Federal em Latossolo Vermelho distrófico, textura argilosa (45\% argila) (Lima et al., 2014). Os frutos foram produzidos em sistema convencional e orgânico e colhidos manualmente diretamente na copa das plantas, tendo como índice de colheita a coloração dos frutos no ponto de maturação com $20 \%$ da cor da casca amarelada. A colheita e transporte dos frutos foram realizados nas primeiras horas do dia com o objetivo de evitar o calor de campo. No Laboratório de Ciência e Tecnologia de Alimentos da Embrapa Cerrados, os frutos foram resfriados em câmara fria $\left(10{ }^{\circ} \mathrm{C} \pm 1{ }^{\circ} \mathrm{C}\right.$ e $80 \%$ de umidade relativa).

O experimento consistiu nos seguintes tratamentos: (i) Frutos de $P$. tenuifila oriundos de produção convencional armazenados sob condição ambiente Convencional Ambiente; (ii) Frutos de P. tenuifila oriundos de produção convencional armazenados a $10{ }^{\circ} \mathrm{C}$ - Convencional $10{ }^{\circ} \mathrm{C}$ (iii) Frutos de P. tenuifila oriundos de produção orgânica armazenados sob condição ambiente - Orgânico Ambiente (iv) Frutos de $P$. tenuifila oriundos de produção orgânica armazenados a $10{ }^{\circ} \mathrm{C}$ - Orgânico $10{ }^{\circ} \mathrm{C}(\mathrm{v})$ Frutos de $P$. tenuifila oriundos de produção convencional acondicionados em embalagem PEAD com $19 \mathrm{~cm} \mathrm{x}$ $29 \mathrm{~cm}$ com $30 \mu \mathrm{m}$ de espessura e armazenados sob condição ambiente - PEAD Convencional Ambiente (vi) Frutos de P. tenuifila oriundos de produção convencional acondicionados em embalagem PEAD com $19 \mathrm{~cm}$ x $29 \mathrm{~cm}$ com $30 \mu \mathrm{m}$ de espessura e armazenados a $10{ }^{\circ} \mathrm{C}$ - PEAD Convencional $10{ }^{\circ} \mathrm{C}$ (vii) Frutos de P. tenuifila oriundos de produção orgânica acondicionados em embalagem PEAD com $19 \mathrm{~cm} \mathrm{x} 29 \mathrm{~cm}$ com $30 \mu \mathrm{m}$ de espessura e armazenados sob condição ambiente - PEAD Orgânico Ambiente (viii) Frutos de P. tenuifila oriundos de produção orgânica acondicionados em embalagem PEAD com $19 \mathrm{~cm}$ x $29 \mathrm{~cm}$ com $30 \mu \mathrm{m}$ de espessura e armazenados a $10{ }^{\circ} \mathrm{C}$ - PEBD Orgânico $10{ }^{\circ} \mathrm{C}$. Os frutos dos diferentes tratamentos foram armazenados sob refrigeração em câmara fria com umidade relativa de $90 \%$ na temperatura de $10{ }^{\circ} \mathrm{C}$. Para a simulação de condição ambiente, os frutos também foram mantidos em câmara fria na temperatura de 23 ${ }^{\circ} \mathrm{C}$ e de $77 \%$ UR. O período de armazenamento para todos os tratamentos foi de 10 dias.
Ao zero, seis e 10 dias de armazenamento, analisouse a perda de massa fresca (PMF) dos frutos obtida pela diferença de peso entre a massa inicial e a massa no momento da avaliação. Textura onde para a análise utilizou-se o texturômetro da marca Brookfield texture Analyzer, modelo CT3 4500. A análise consistiu no teste de resistência de perfuração (teste normal), sendo adotados os padrões de Trigger (força): $10 \mathrm{~g}$, Deformation (deformação): $10 \mathrm{~mm}$ e Speed (velocidade): $10 \mathrm{~mm} / \mathrm{s}$ com o auxílio da ponteira TA 17 Cone $30 \mathrm{~mm} \mathrm{D}, 45^{\circ}$. Os resultados foram apresentados em Newton (N). Concentração gasosa no interior das embalagens, sendo que as análises da concentração de $\mathrm{O}_{2}$ e $\mathrm{CO}_{2}$ no interior das embalagens foram realizadas utilizando-se o equipamento CheckPoint II (PBI-Dansensor America Inc) de acordo com Rinaldi et al. (2009). Cor $\left(L^{*}, a^{*}, b^{*}\right)$ determinada em espectrofotômetro MiniScan ${ }^{\circledR}$ EZ marca HunterLab, sendo realizadas cinco leituras por fruto. $\mathrm{O}$ valor de $\mathrm{L}^{*}$ define a luminosidade $\left(\mathrm{L}^{*}=0\right.$ preto e $\mathrm{L}^{*}=100$ branco) e $\mathrm{a}^{*} \mathrm{e}^{*}$ são responsáveis pela cromaticidade $\left(+a^{*}\right.$ vermelho $e-a^{*}$ verde $), b^{*}\left(+b^{*}\right.$ amarelo e $-b^{*}$ azul). Por meio do módulo $\mathrm{L}^{*}, \mathrm{a}^{*}$ e $\mathrm{b}^{*}$ foi possível calcular o incremento no escurecimento $\left[\left(\left(L^{*}-L^{*} 0\right)^{2}+\left(a^{*}-a^{*} 0\right)^{2}+\left(b^{*}-b^{*} 0\right)^{2}\right)^{1 / 2}\right]$, chroma (saturação ou intensidade da cor; 0 - cor impura e 60 - cor pura) e o ângulo hue (ângulo da cor; $0^{\circ}$ vermelho; $90^{\circ}$ amarelo; $180^{\circ}$ verde; $270^{\circ}$ azul e $360^{\circ}$ negro) por meio das fórmulas: chroma $\left[\left(\mathrm{a}^{2}+\mathrm{b}^{2}\right)^{1 / 2}\right]$ e ângulo hue [arco tangente (b/a)] para $\mathrm{a}^{*}$ positivo e [arco tangente $\left(\mathrm{b}^{*} / \mathrm{a}^{*}\right)(-1)+90$ ] para $\mathrm{a}^{*}$ negativo, conforme recomendado por Hunterlab (2008). Análises de pH, acidez titulável, sólidos solúveis e Ratio realizadas de acordo com a Association of Official Analytical (2016). Para as análises de $\mathrm{pH}$, acidez titulável e sólidos solúveis os frutos de $P$. tenuifila foram triturados integralmente em liquidificador industrial formando uma massa base conforme POP CPAC-2012.

$\mathrm{O}$ experimento foi realizado em delineamento experimental inteiramente casualizado em esquema fatorial 2 (ambiente e refrigerado) x 3 (tratamentos) $\mathrm{x}$ 3 (dias de avaliação) com três repetições para cada tratamento, sendo que cada repetição consistiu em seis frutos. Para as análises de cor foram utilizados os seis frutos por tratamento sendo realizadas cinco leituras por fruto em cada dia de análise. Para textura foram realizadas três leituras em cada um dos seis frutos 
analisados. Os dados foram submetidos à análise de variância utilizando o teste $\mathrm{F}$ e as médias foram comparadas entre si pelo teste Tukey a $5 \%$ de probabilidade de erro. Todas as análises estatísticas foram realizadas com o auxílio do programa estatístico Sisvar (Ferreira, 2019).

\section{Resultados e Discussão}

Frutos de $P$. tenuifila produzidos em sistema convencional e orgânico não apresentaram variação espécie produzidos em sistema convencional.

A aparência dos frutos de maracujá é significativamente afetada pela perda de massa fresca ocasionando em prejuízos na comercialização in natura, considerando que os consumidores adquirem os frutos de acordo com a aparência (Solino et al., 2012). Os frutos de $P$. tenuifila possuem casca com espessura muito fina resultando no ressecamento da mesma com a perda de massa fresca. Além disso, os frutos de maracujá são comercializados por peso, resultando em perdas econômicas para o produtor significativa na vida útil pós-colheita. Os frutos desta espécie quando armazenados na temperatura de $10{ }^{\circ} \mathrm{C}$ tiveram maior durabilidade pós-colheita. A embalagem PEBD também foi efetiva no aumento da vida útil pós-colheita dos frutos de $P$. tenuifila. Quanto à perda de massa fresca, os frutos produzidos em sistema convencional, acondicionados em embalagem PEBD e armazenados na temperatura de $10{ }^{\circ} \mathrm{C}$ apresentaram menores valores atingindo o valor de $1,02 \%$ aos 10 dias de armazenamento. Os resultados comprovam que a junção da embalagem adequada e a redução da temperatura de armazenamento foram responsáveis pela manutenção da vida útil pós-colheita dos frutos desta espécie (Tabela 1). O resultado também corrobora com Kader (2010) que afirma que a perda de massa fresca dos produtos vegetais é fortemente reduzida por meio do uso de filmes plásticos flexíveis. Nos frutos sem embalagem, nas duas condições de cultivo, ocorreu menor perda de massa fresca quando armazenados em condição de refrigeração na temperatura de $10{ }^{\circ} \mathrm{C}$. Frutos produzidos em sistema orgânico sem embalagem, armazenados em condição ambiente apresentaram a maior perda de massa fresca atingindo 34,77\% aos 10 dias de armazenamento. Em todos os tratamentos, frutos produzidos neste sistema também apresentaram maior percentagem de perda de massa fresca quando comparado aos frutos da mesma
Tabela 1. Valores médios de perda de massa fresca (PMF) e textura dos frutos, oxigênio (\%) e dióxido de carbono (\%) no interior das embalagens com frutos de Passiflora tenuifila acondicionados em embalagem de PEAD $30 \mu \mathrm{m}$ a armazenados por 10 dias em condição ambiente e refrigerada

\begin{tabular}{|c|c|c|c|}
\hline \multicolumn{4}{|c|}{ Perda de Massa Fresca (\%) } \\
\hline \multicolumn{4}{|c|}{ Tempo de Armazenamento (dias) } \\
\hline Tratamentos & zero & 6 & 10 \\
\hline Convencional Ambiente & $0,00 \mathrm{aC}$ & $19,21 \mathrm{bB}$ & $27,16 \mathrm{bA}$ \\
\hline Convencional $10^{\circ} \mathrm{C}$ & $0,00 \mathrm{aB}$ & $8,99 \mathrm{cA}$ & $10,29 \mathrm{dA}$ \\
\hline Orgânico Ambiente & $0,00 \mathrm{aC}$ & $27,52 \mathrm{aB}$ & $34,77 \mathrm{aA}$ \\
\hline Orgânico $10^{\circ} \mathrm{C}$ & $0,00 \mathrm{aB}$ & $16,27 \mathrm{bA}$ & $20,98 \mathrm{cA}$ \\
\hline PEAD Convencional Ambiente & $0,00 \mathrm{aB}$ & $0,67 \mathrm{~dB}$ & $7,90 \mathrm{dA}$ \\
\hline PEAD Convencional $10^{\circ} \mathrm{C}$ & $0,00 \mathrm{aA}$ & $0,71 \mathrm{dA}$ & $1,02 \mathrm{eA}$ \\
\hline PEAD Orgânico Ambiente & $0,00 \mathrm{aB}$ & $10,70 \mathrm{cA}$ & $10,75 \mathrm{dA}$ \\
\hline PEAD Orgânico $10^{\circ} \mathrm{C}$ & $0,00 \mathrm{aB}$ & $0,98 \mathrm{~dB}$ & $7,41 \mathrm{dA}$ \\
\hline \multicolumn{4}{|c|}{ Textura (N) } \\
\hline Convencional Ambiente & $1,07 \mathrm{aA}$ & $0,83 \quad \mathrm{bA}$ & 1,00 abcA \\
\hline Convencional $10{ }^{\circ} \mathrm{C}$ & $1,07 \mathrm{aA}$ & $1,27 \mathrm{abA}$ & 1,07 abcA \\
\hline Orgânico Ambiente & $1,23 \mathrm{aA}$ & $0,80 \quad b B$ & $0,90 \mathrm{abcAB}$ \\
\hline Orgânico $10^{\circ} \mathrm{C}$ & $1,23 \mathrm{aA}$ & 1,40 aA & $1,20 \quad a b A$ \\
\hline PEAD Convencional Ambiente & $1,07 \mathrm{aA}$ & $0,93 \mathrm{abA}$ & 0,76 \\
\hline PEAD Convencional $10^{\circ} \mathrm{C}$ & $1,07 \mathrm{aA}$ & $1,13 \mathrm{abA}$ & 1,33 \\
\hline PEAD Orgânico Ambiente & $1,23 \mathrm{aA}$ & $0,90 \mathrm{bAB}$ & $0,70 \quad \mathrm{cB}$ \\
\hline PEAD Orgânico $10^{\circ} \mathrm{C}$ & $1,23 \mathrm{aA}$ & $1,17 \mathrm{abA}$ & 1,67 abcA \\
\hline \multicolumn{4}{|c|}{ Oxigênio (\%) } \\
\hline PEAD Convencional Ambiente & $20,95 \mathrm{aA}$ & $8,57 \mathrm{bC}$ & $13,07 \mathrm{cB}$ \\
\hline PEAD Convencional $10^{\circ} \mathrm{C}$ & $20,95 \mathrm{aA}$ & $10,90 \mathrm{abC}$ & $16,87 \mathrm{abB}$ \\
\hline PEAD Orgânico Ambiente & $20,95 \mathrm{aA}$ & $11,70 \mathrm{abB}$ & $14,43 \mathrm{bcB}$ \\
\hline PEAD Orgânico $10^{\circ} \mathrm{C}$ & $20,95 \mathrm{aA}$ & $14,10 \quad a B$ & 19,43 aA \\
\hline \multicolumn{4}{|c|}{ Dióxido de Carbono (\%) } \\
\hline PEAD Convencional Ambiente & $0,04 \mathrm{aC}$ & 6,00 aA & $4,63 \mathrm{aB}$ \\
\hline PEAD Convencional $10^{\circ} \mathrm{C}$ & $0,04 \mathrm{aC}$ & 4,90 aA & $2,93 \mathrm{bB}$ \\
\hline PEAD Orgânico Ambiente & $0,04 \mathrm{aB}$ & 5,97 aA & 4,80 aA \\
\hline PEAD Orgânico $10^{\circ} \mathrm{C}$ & $0,04 \mathrm{aC}$ & 4,73 aA & $2,17 \mathrm{bB}$ \\
\hline
\end{tabular}

Letras minúsculas iguais, na mesma coluna, não diferem significativamente ao nível de $5 \%$ pelo teste Tukey.

Letras maiúsculas iguais, na mesma linha, não diferem significativamente ao nível de $5 \%$ pelo teste Tukey. 
(Pereira et al., 2018). Os frutos de maracujá são climatéricos havendo aumento da taxa respiratória e da emissão de etileno, causando a rápida senescência após a colheita (Silva, Silva e Souza, 2019).

Para os frutos de P. tenuifila é recomendado o manuseio de forma a evitar o empilhamento devido as suas características físicas de não suportar peso. Também apresentam desidratação e enrugamento da casca, o que não é desejado na comercialização.

Portanto, a definição da forma mais adequada de manuseio e armazenamento dos frutos desta espécie é imprescindível. Em frutos de maracujá azedo, Passiflora setacea, Passiflora alata e Passiflora cincinnata, baixas temperaturas são fundamentais na redução da perda de massa fresca (Rinaldi et al., 2017a e 2017b; Rinaldi et al., 2019a e 2019b; Rinaldi, Dianese e Costa, 2021).

Os tratamentos em que os frutos foram produzidos em sistema convencional e acondicionados em embalagem PEAD armazenados sob condição ambiente e refrigerado e, também, os frutos produzidos em sistema orgânico acondicionados em embalagem PEAD armazenados a $10{ }^{\circ} \mathrm{C}$ apresentaram perda de massa fresca inferior a $8 \%$ durante os 10 dias de armazenamento, o que é desejável. Assim, se for considerado o padrão utilizado para maracujazeiroazedo, esses tratamentos são recomendados para $P$. tenuifila, uma vez que frutos de maracujazeiro azedo são considerados murchos a partir de uma perda de $8 \%$ do seu peso inicial, por prejudicar a aparência do fruto, depreciando o seu valor comercial (FAEP, 2008).

A textura dos frutos submetidos aos diferentes tratamentos variou entre 0,70 a $1,67 \mathrm{~N}$ sendo considerados valores muito baixos quando comparado a outros frutos de maracujá como $P$. cincinnata (Rinaldi, Dianese e Costa, 2021) e P. alata (Rinaldi et al., 2019a e 2019b). Durante os 10 dias de armazenamento ocorreu redução significativa para esta variável nos tratamentos com os frutos produzidos no sistema orgânico apenas armazenado a $10{ }^{\circ} \mathrm{C}$, e no mesmo sistema acondicionado em embalagem PEAD armazenado em condição ambiente (Tabela 1). A não redução de textura dos frutos é desejável e pode ocorrer devido a composição da casca dos frutos. A diminuição da textura ocorre devido à solubilização de pectinas e hidrólise de polissacarídeos estruturais da parede celular (D’Abadia et al., 2020).
A maior redução na concentração de oxigênio no interior das embalagens contendo os frutos de $P$. tenuifila ocorreu até os seis dias de armazenamento, sendo mais acentuado na embalagem contendo os frutos produzidos em sistema convencional, e armazenados sob condição ambiente atingindo o valor de $8,57 \%$. Do sexto ao décimo dia de armazenamento os valores de oxigênio aumentaram em todos os tratamentos, com exceção, do PEAD Orgânico Ambiente (Tabela 1). De maneira geral, a embalagem não foi eficiente na manutenção da concentração reduzida desejável de oxigênio em seu interior (Floros e Matsos, 2005) devido às características de permeabilidade da mesma a este gás. Mesmo assim, auxiliou na manutenção da vida útil dos frutos da espécie.

Quanto à concentração de dióxido de carbono a maior concentração $(6,00 \%)$ também ocorreu na embalagem em que os frutos foram submetidos ao tratamento Convencional Ambiente aos seis dias de armazenamento. No décimo dia de armazenamento, os valores foram significativamente inferiores aos demais dias de armazenamento com exceção da concentração de dióxido de carbono obtida no tratamento PEAD Orgânico Ambiente que não apresentou valores significativamente superiores ao obtido no sexto dia de armazenamento. Aos 10 dias de armazenamento, a condição ambiente interferiu em maior grau nos níveis de $\mathrm{CO}_{2}$ dos frutos, apresentando maiores concentrações dessa variável na embalagem avaliada devido a maior taxa respiratória dos frutos (Tabela 1).

Não houve diferença na luminosidade dos frutos de P. tenuifila produzidos em sistema convencional e orgânico sendo que a luminosidade (Tabela 2) oscilou entre 45,33 e 66,50 com a tendência, na maioria dos tratamentos, de aumento dos valores durante o armazenamento. Maiores valores de luminosidade indicam que os produtos são mais claros (AMSAM, 2012) $\left(\mathrm{L}^{*}=0\right.$ preto e $\mathrm{L}^{*}=100$ branco). Durante o armazenamento, as variações ocorreram nos frutos produzidos no sistema orgânico armazenado a $10{ }^{\circ} \mathrm{C}$ apresentando escurecimento dos frutos aos seis e 10 dias de armazenamento em relação à matéria-prima (dia zero). Nos frutos produzidos em sistema convencional acondicionado em embalagem PEAD e armazenados em condição ambiente aos seis dias de 
armazenamento, os valores de luminosidade foram superiores aos do dia zero. Frutos produzidos no sistema orgânico acondicionado no mesmo tipo de embalagem e armazenamento em condição ambiente estavam mais claros aos 10 dias de armazenamento, corroborando com Botelho et al. (2019) que afirmam que ao longo do amadurecimento ocorre à mudança de cor da casca dos frutos devido às transformações fisiológicas que também alteram as suas características físicoquímicas.

De acordo com os valores de $\mathrm{a}^{*}, \mathrm{em}$ todos os tratamentos ocorreu alteração da cor da casca dos frutos de levemente verdes para vermelho $\left(+\mathrm{a}^{*}\right.$ vermelho e $a^{*}$ verde) (Tabela 2$)$. A maior variação nos valores de $\mathrm{a}^{*}$ ocorreram nos frutos armazenados sob condição ambiente, $o$ que já era esperado, uma vez que em condição ambiente a maior atividade metabólica nos frutos influenciou na alteração da cor.

Os valores de $b^{*}$ estiveram na faixa de cor amarelo $\left(+b^{*}\right.$ amarelo e $-b^{*}$ azul $)$ não apresentando valores negativos, com variação significativa apenas nos tratamentos em que os frutos foram armazenados sob condição ambiente (Tabela 2).

$\mathrm{O}$ incremento no escurecimento variou entre zero e 53,15 sendo considerado um valor alto para esta variável. Com exceção dos frutos produzidos em sistema convencional armazenados em condição ambiente, que não apresentaram aumento significativo entre os seis e 10 dias, todos os demais tratamentos apresentaram incremento no escurecimento significativo durante os 10 dias de armazenamento (Tabela 2). Os resultados comprovam que a temperatura de armazenamento influência diretamente na coloração dos frutos. $\mathrm{O}$ objetivo de realizar a medida do incremento no escurecimento é verificar
Tabela 2. Valores médios de luminosidade ( $\left.\mathrm{L}^{*}\right), \mathrm{a}^{*}, \mathrm{~b}^{*}$, Incremento no escurecimento (IE) e croma em frutos de Passiflora tenuifila submetidos a diferentes tratamentos

\begin{tabular}{|c|c|c|c|}
\hline \multicolumn{4}{|c|}{ Luminosidade $\left(\mathrm{L}^{*}\right)$} \\
\hline \multicolumn{4}{|c|}{ Tempo de Armazenamento (dias) } \\
\hline Tratamentos & zero & 6 & 10 \\
\hline Convencional Ambiente & $54,99 \mathrm{aA}$ & $54,91 \mathrm{abcA}$ & $57,35 \mathrm{abA}$ \\
\hline Convencional $10{ }^{\circ} \mathrm{C}$ & 54,99 aA & 49,39 bcA & $50,48 \mathrm{bcA}$ \\
\hline Orgânico Ambiente & $57,42 \mathrm{aA}$ & $56,45 \mathrm{abcA}$ & $56,79 \mathrm{abA}$ \\
\hline Orgânico $10^{\circ} \mathrm{C}$ & $57,42 \mathrm{aA}$ & $48,02 \quad \mathrm{cB}$ & $45,33 \mathrm{cB}$ \\
\hline PEAD Convencional Ambiente & $54,99 \mathrm{aB}$ & 64,14 aA & $62,86 \mathrm{aAB}$ \\
\hline PEAD Convencional $10^{\circ} \mathrm{C}$ & $54,99 \mathrm{aA}$ & $55,78 \mathrm{abcA}$ & $60,75 \mathrm{abA}$ \\
\hline PEAD Orgânico Ambiente & $57,42 \mathrm{aB}$ & $61,68 \mathrm{aAB}$ & 66,50 aA \\
\hline PEAD Orgânico $10^{\circ} \mathrm{C}$ & $57,42 \mathrm{aA}$ & 59,92 abA & $58,98 \mathrm{abA}$ \\
\hline \multicolumn{4}{|c|}{$\mathbf{a}^{*}$} \\
\hline Convencional Ambiente & $0,67 \mathrm{aB}$ & 16,17 aA & $16,88 \mathrm{aA}$ \\
\hline Convencional $10{ }^{\circ} \mathrm{C}$ & $0,67 \mathrm{aB}$ & $4,05 \quad \mathrm{cA}$ & $3,95 \mathrm{bA}$ \\
\hline Orgânico Ambiente & $0,39 \mathrm{aB}$ & 15,61 aA & $16,77 \mathrm{aA}$ \\
\hline Orgânico $10^{\circ} \mathrm{C}$ & $0,39 \mathrm{aB}$ & $3,56 \mathrm{cA}$ & $4,02 \mathrm{bA}$ \\
\hline PEAD Convencional Ambiente & $0,67 \mathrm{aC}$ & $12,17 \quad b B$ & $15,57 \mathrm{aA}$ \\
\hline PEAD Convencional $10^{\circ} \mathrm{C}$ & $0,67 \mathrm{aB}$ & $4,02 \quad \mathrm{cA}$ & $5,20 \mathrm{bA}$ \\
\hline PEAD Orgânico Ambiente & $0,39 \mathrm{aC}$ & 9,49 bB & $16,43 \mathrm{aA}$ \\
\hline PEAD Orgânico $10^{\circ} \mathrm{C}$ & $0,39 \mathrm{aB}$ & $1,83 \mathrm{cAB}$ & $2,98 \mathrm{bA}$ \\
\hline \multicolumn{4}{|c|}{$\mathbf{b}^{*}$} \\
\hline Convencional Ambiente & $36,89 \mathrm{aB}$ & $44,01 \mathrm{abAB}$ & $45,33 \mathrm{abcA}$ \\
\hline Convencional $10{ }^{\circ} \mathrm{C}$ & $36,89 \mathrm{aA}$ & $35,44 \quad \mathrm{bA}$ & $36,77 \quad \mathrm{cA}$ \\
\hline Orgânico Ambiente & $38,55 \mathrm{aB}$ & 46,50 & 47,10 abA \\
\hline Orgânico $10{ }^{\circ} \mathrm{C}$ & $38,55 \mathrm{aA}$ & 35,57 & $36,02 \quad \mathrm{cA}$ \\
\hline PEAD Convencional Ambiente & $36,89 \mathrm{aB}$ & $48,28 \quad \mathrm{aA}$ & 50,00 abA \\
\hline PEAD Convencional $10^{\circ} \mathrm{C}$ & $36,89 \mathrm{aA}$ & 39,28 abA & 42,96 bcA \\
\hline PEAD Orgânico Ambiente & $38,55 \mathrm{aB}$ & $46,12 \quad \mathrm{aA}$ & 53,23 aA \\
\hline PEAD Orgânico $10^{\circ} \mathrm{C}$ & $38,55 \mathrm{aA}$ & $42,25 \mathrm{abA}$ & $43,20 \mathrm{bcA}$ \\
\hline
\end{tabular}

\begin{tabular}{|c|c|c|c|}
\hline \multicolumn{4}{|c|}{ Incremento no escurecimento (IE) } \\
\hline Convencional Ambiente & $0,00 \mathrm{aB}$ & 26,99 aA & $27,94 \quad \mathrm{cA}$ \\
\hline Convencional $10{ }^{\circ} \mathrm{C}$ & $0,00 \mathrm{aC}$ & $15,86 \mathrm{aB}$ & $36,95 \mathrm{abcA}$ \\
\hline Orgânico Ambiente & $0,00 \mathrm{aC}$ & $25,81 \mathrm{aB}$ & 35,21 bcA \\
\hline Orgânico $10^{\circ} \mathrm{C}$ & $0,00 \mathrm{aC}$ & $20,62 \mathrm{aB}$ & $45,42 \mathrm{abA}$ \\
\hline PEAD Convencional Ambiente & $0,00 \mathrm{aC}$ & $23,37 \mathrm{aB}$ & $48,63 \mathrm{abA}$ \\
\hline PEAD Convencional $10^{\circ} \mathrm{C}$ & $0,00 \mathrm{aC}$ & $12,84 \mathrm{aB}$ & 53,15 aA \\
\hline PEAD Orgânico Ambiente & $0,00 \mathrm{aC}$ & $25,90 \mathrm{aB}$ & 48,55 abA \\
\hline PEAD Orgânico $10^{\circ} \mathrm{C}$ & $0,00 \mathrm{aC}$ & $14,10 \mathrm{aB}$ & $46,06 \mathrm{abA}$ \\
\hline \multicolumn{4}{|c|}{ Croma } \\
\hline Convencional Ambiente & $37,05 \mathrm{aB}$ & $47,18 \mathrm{abA}$ & 48,49 abA \\
\hline Convencional $10{ }^{\circ} \mathrm{C}$ & $37,05 \mathrm{aA}$ & $35,98 \quad \mathrm{cA}$ & $37,19 \quad \mathrm{cA}$ \\
\hline Orgânico Ambiente & $38,72 \mathrm{aB}$ & 49,23 & $50,13 \mathrm{abA}$ \\
\hline Orgânico $10^{\circ} \mathrm{C}$ & $38,72 \mathrm{aA}$ & 36,25 & $36,49 \quad \mathrm{cA}$ \\
\hline PEAD Convencional Ambiente & $37,05 \mathrm{aB}$ & 49,93 & 52,44 abA \\
\hline PEAD Convencional $10^{\circ} \mathrm{C}$ & $37,05 \mathrm{aA}$ & 39,75 bcA & 43,42 bcA \\
\hline PEAD Orgânico Ambiente & $38,72 \mathrm{aC}$ & 47,17 abB & 55,75 aA \\
\hline PEAD Orgânico $10^{\circ} \mathrm{C}$ & $38,72 \mathrm{aA}$ & $42,25 \mathrm{abcA}$ & $43,55 \mathrm{bcA}$ \\
\hline
\end{tabular}

Letras minúsculas iguais, na mesma coluna, não diferem significativamente ao nível de $5 \%$ pelo teste Tukey.

Letras maiúsculas iguais, na mesma linha, não diferem significativamente ao nível de $5 \%$ pelo teste Tukey. 
o escurecimento do produto durante o armazenamento. De acordo com Rinaldi et al. (2017a) para produtos vegetais, considera-se que, quando os valores de $\mathrm{E}>10$, o grau de escurecimento é elevado não sendo desejável para o produto. Visualmente não foi observado escurecimento significativo nos frutos de $P$. tenuifila nos diferentes tratamentos avaliados. Assim, essa variável pode não ser adequada para a avaliação de frutos dessa espécie.

A cromaticidade foi mais uniforme nos frutos armazenados na temperatura de $10{ }^{\circ} \mathrm{C}$ não havendo diferença no decorrer do armazenamento (Tabela 2). Nos tratamentos armazenados sob condição ambiente, com exceção dos frutos produzidos em sistema orgânico acondicionados na embalagem PEAD, que apresentaram variação durante os 10 dias de armazenamento, nos demais tratamentos, só variou aos seis e 10 dias em relação ao início do armazenamento (dia zero). O chroma ou cromaticidade expressa a intensidade da cor, ou seja, a cor em termos de saturação de pigmentos. O chroma indica a intensidade e saturação da cor (D'Abadia et al., 2020). Os maiores valores de chroma foram apresentados pelos frutos armazenados em condição ambiente, comprovando que para frutos de $P$. tenuifila esta variável é influenciada pela temperatura de armazenamento.

Quando armazenados em temperatura ambiente a cor dos frutos de $P$. tenuifila foi mais avermelhado em todos os tratamentos até os seis dias de armazenamento. Aos 10 dias, frutos produzidos em sistema orgânico apenas armazenado a $10^{\circ} \mathrm{C}$, e os produzidos em sistema orgânico e convencional acondicionados em embalagem PEAD e armazenados a $10{ }^{\circ} \mathrm{C}$ apresentaram valores de ângulo hue inferior aos demais dias de armazenamento (Tabela 3).
Tabela 3. Valores médios de ângulo hue, $\mathrm{pH}$, acidez titulável, sólidos solúveis e Ratio em frutos de Passiflora tenuifila submetidos a diferentes tratamentos

\begin{tabular}{|c|c|c|c|}
\hline \multicolumn{4}{|c|}{ Ângulo hue } \\
\hline \multicolumn{4}{|c|}{ Tempo de Armazenamento (dias) } \\
\hline Tratamentos & zero & 6 & 10 \\
\hline Convencional Ambiente & $85,51 \mathrm{aA}$ & $67,07 \mathrm{aA}$ & $68,65 \mathrm{abA}$ \\
\hline Convencional $10{ }^{\circ} \mathrm{C}$ & $85,51 \mathrm{aA}$ & $85,49 \mathrm{aA}$ & 70,27 aA \\
\hline Orgânico Ambiente & 89,75 aA & $77,27 \mathrm{aA}$ & $69,40 \mathrm{abA}$ \\
\hline Orgânico $10^{\circ} \mathrm{C}$ & $89,75 \mathrm{aA}$ & $84,44 \mathrm{aA}$ & $39,84 \mathrm{cB}$ \\
\hline PEAD Convencional Ambiente & $85,51 \mathrm{aA}$ & $79,03 \mathrm{aA}$ & 72,78 aA \\
\hline PEAD Convencional $10{ }^{\circ} \mathrm{C}$ & $85,51 \mathrm{aA}$ & $84,96 \mathrm{aA}$ & $59,61 \mathrm{abcB}$ \\
\hline PEAD Orgânico Ambiente & $89,75 \mathrm{aA}$ & $80,19 \mathrm{aA}$ & 72,83 aA \\
\hline PEBD Orgânico $10^{\circ} \mathrm{C}$ & $89,75 \mathrm{aA}$ & $87,75 \mathrm{aA}$ & $41,84 \mathrm{bcB}$ \\
\hline \multicolumn{4}{|c|}{$\mathrm{pH}$} \\
\hline Convencional Ambiente & $5,20 \mathrm{aC}$ & $5,94 \mathrm{aA}$ & $5,49 \mathrm{aB}$ \\
\hline Convencional $10{ }^{\circ} \mathrm{C}$ & $5,20 \mathrm{aC}$ & $5,90 \mathrm{aA}$ & $5,44 \mathrm{aB}$ \\
\hline Orgânico Ambiente & $5,19 \mathrm{aC}$ & $5,93 \mathrm{aA}$ & $5,47 \mathrm{aB}$ \\
\hline Orgânico $10^{\circ} \mathrm{C}$ & $5,19 \mathrm{aC}$ & $5,76 \mathrm{aA}$ & $5,46 \mathrm{aB}$ \\
\hline PEAD Convencional Ambiente & $5,20 \mathrm{aC}$ & $6,00 \mathrm{aA}$ & $5,57 \mathrm{aB}$ \\
\hline PEAD Convencional $10{ }^{\circ} \mathrm{C}$ & $5,20 \mathrm{aA}$ & $5,35 \mathrm{bA}$ & $5,39 \mathrm{aA}$ \\
\hline PEAD Orgânico Ambiente & $5,19 \mathrm{aB}$ & $6,04 \mathrm{aA}$ & $5,26 \mathrm{aB}$ \\
\hline PEAD Orgânico $10^{\circ} \mathrm{C}$ & 5,19 aA & $5,26 \mathrm{bA}$ & $5,41 \mathrm{aA}$ \\
\hline \multicolumn{4}{|c|}{ Acidez Titulável (\%) } \\
\hline Convencional Ambiente & $0,13 \mathrm{aA}$ & $0,13 \mathrm{aA}$ & $0,16 \mathrm{aA}$ \\
\hline Convencional $10^{\circ} \mathrm{C}$ & $0,13 \mathrm{aA}$ & $0,11 \mathrm{aA}$ & $0,14 \mathrm{aA}$ \\
\hline Orgânico Ambiente & $0,32 \mathrm{aA}$ & $0,09 \mathrm{aA}$ & $0,12 \mathrm{aA}$ \\
\hline Orgânico $10^{\circ} \mathrm{C}$ & $0,32 \mathrm{aA}$ & $0,10 \mathrm{aA}$ & $0,11 \mathrm{aA}$ \\
\hline PEAD Convencional Ambiente & $0,13 \mathrm{aA}$ & $0,13 \mathrm{aA}$ & $0,12 \mathrm{aA}$ \\
\hline PEAD Convencional $10{ }^{\circ} \mathrm{C}$ & $0,13 \mathrm{aA}$ & $0,14 \mathrm{aA}$ & $0,12 \mathrm{aA}$ \\
\hline PEAD Orgânico Ambiente & $0,32 \mathrm{aA}$ & $0,07 \mathrm{aA}$ & $0,10 \mathrm{aA}$ \\
\hline PEAD Orgânico $10^{\circ} \mathrm{C}$ & $0,32 \mathrm{aA}$ & $0,12 \mathrm{aA}$ & $0,12 \mathrm{aA}$ \\
\hline \multicolumn{4}{|c|}{ Sólidos Solúveis $\left({ }^{\circ} \mathrm{Brix}\right)$} \\
\hline Convencional Ambiente & $5,90 \mathrm{aB}$ & $6,23 \mathrm{aAB}$ & $6,83 \mathrm{abA}$ \\
\hline Convencional $10^{\circ} \mathrm{C}$ & $5,90 \mathrm{aB}$ & $6,33 \mathrm{aAB}$ & $7,10 \quad \mathrm{aA}$ \\
\hline Orgânico Ambiente & $6,47 \mathrm{aA}$ & $5,50 \mathrm{abB}$ & 6,70 abA \\
\hline Orgânico $10{ }^{\circ} \mathrm{C}$ & $6,47 \mathrm{aA}$ & 6,40 aA & $6,40 \mathrm{abcA}$ \\
\hline PEAD Convencional Ambiente & $5,90 \mathrm{aA}$ & $4,50 \mathrm{bB}$ & $5,23 \mathrm{cdAB}$ \\
\hline PEAD Convencional $10{ }^{\circ} \mathrm{C}$ & $5,90 \mathrm{aA}$ & 6,37 aA & $5,67 \mathrm{bcdA}$ \\
\hline PEAD Orgânico Ambiente & $6,47 \mathrm{aA}$ & $4,77 \mathrm{bB}$ & $4,97 \quad \mathrm{~dB}$ \\
\hline PEAD Orgânico $10^{\circ} \mathrm{C}$ & 6,47 aA & $6,10 \mathrm{aAB}$ & $5,30 \mathrm{cdB}$ \\
\hline \multicolumn{4}{|c|}{ Ratio } \\
\hline Convencional Ambiente & $45,38 \mathrm{aA}$ & $47,92 \mathrm{cbA}$ & 42,69 bA \\
\hline Convencional $10^{\circ} \mathrm{C}$ & $45,38 \mathrm{aB}$ & $57,54 \mathrm{bA}$ & $50,7 \mathrm{abA}$ \\
\hline Orgânico Ambiente & $20,22 \mathrm{bB}$ & $61,11 \mathrm{aA}$ & 55,83 aA \\
\hline Orgânico $10^{\circ} \mathrm{C}$ & $20,22 \mathrm{bB}$ & 64,00 aA & 58,18 aA \\
\hline PEAD Convencional Ambiente & $45,38 \mathrm{aA}$ & $34,61 \mathrm{~dB}$ & 43,58 bA \\
\hline PEAD Convencional $10^{\circ} \mathrm{C}$ & $45,38 \mathrm{aA}$ & $45,50 \mathrm{cbA}$ & $47,25 \mathrm{bA}$ \\
\hline PEAD Orgânico Ambiente & $20,22 \mathrm{bC}$ & 68,14 aA & $49,70 \mathrm{abB}$ \\
\hline PEAD Orgânico $10^{\circ} \mathrm{C}$ & $20,22 \mathrm{bB}$ & $50,83 \mathrm{bA}$ & $44,17 \mathrm{bA}$ \\
\hline
\end{tabular}

Letras minúsculas iguais, na mesma coluna, não diferem significativamente ao nível de 5\% pelo teste Tukey. Letras maiúsculas iguais, na mesma linha, não diferem significativamente ao nível de $5 \%$ pelo teste Tukey. 
Durante todo o período de armazenamento os valores situaram-se no primeiro quadrante (zero a $90^{\circ}$ ), ou seja, coloração variando do vermelho ao amarelo. O ângulo hue define a tonalidade da cor e pode indicar o grau de maturação do fruto.

Os valores de $\mathrm{pH}$ estiveram entre 5,19 e 6,04 durante os 10 dias de armazenamento, não havendo diferença nos tratamentos em que os frutos foram produzidos em sistema convencional e orgânico e acondicionados em embalagem PEAD armazenados na temperatura de $10{ }^{\circ} \mathrm{C}$. Holanda et al. (2015) obtiveram valores inferiores $(4,43)$ de $\mathrm{pH}$ em frutos de $P$. tenuifila. Nos demais tratamentos, com exceção do PEAD Orgânico Ambiente, ocorreu aumento aos seis e 10 dias em relação ao dia zero, com maiores valores de $\mathrm{pH}$ aos seis dias. Estes dois tratamentos também apresentaram menores valores de $\mathrm{pH}$ em relação aos demais aos seis dias. No tratamento PEAD Orgânico Ambiente o pH aos 10 dias de armazenamento não diferiu do início do armazenamento (dia zero) (Tabela 3).

Os diferentes tratamentos não influenciaram na acidez titulável dos frutos de $P$. tenuifila durante todo o experimento onde os valores foram entre $0,07 \% \mathrm{e}$ $0,32 \%$. Acidez titulável superior $(0,41 \%)$ foram obtidos por Silveira et al. (2019) na polpa de frutos de $P$. tenuifila. Na mesma espécie Holanda (2015) obteve valor médio de $0,19 \%$ estando na faixa de valores obtido no presente trabalho.

Quanto aos sólidos solúveis os frutos submetidos aos tratamentos Orgânico $10{ }^{\circ} \mathrm{C}$ e PEAD Convencional $10{ }^{\circ} \mathrm{C}$ não foram influenciados para esta variável. Os valores de sólidos solúveis foram de 4,50 ${ }^{\circ}$ Brix e $7,10^{\circ}$ Brix sendo bem inferior aos obtidos (22 ${ }^{\circ}$ Brix) por Silveira et al. (2019) na polpa de frutos da mesma espécie. No presente trabalho a variável foi determinada na massa base, ou seja, todo o fruto (polpa, semente e casca) devido ao fruto poder ser consumido em sua totalidade, justificando assim a diferença nos valores. De acordo com Ramos et al. (2013) os sólidos solúveis podem ser influenciados, principalmente, por características genéticas do material e, também, pelas condições do processo produtivo, como adubação, temperatura e disponibilidade de água. Ainda, de acordo com os mesmos autores, os sólidos solúveis é o principal responsável pelo sabor do fruto justificando a sua importância na qualidade pós-colheita.

Os valores de Ratio variaram entre 20,22 e 68,14 durante os 10 dias de armazenamento (Tabela 3 ). Frutos produzidos em sistema convencional armazenado sob condição ambiente e também no tratamento PEAD Convencional $10{ }^{\circ} \mathrm{C}$ não apresentaram diferença nos valores de Ratio. Nos demais tratamentos os valores oscilaram correspondendo ao ocorrido com os sólidos solúveis e acidez titulável. No início do armazenamento, os frutos produzidos em sistema orgânico apresentaram valores de Ratio inferiores aos produzidos em sistema convencional. No entanto, no decorrer do armazenamento os valores foram superiores ou semelhantes aos frutos produzidos em sistema convencional. De acordo com Moura et al. (2016) o Ratio pode ser influenciado pela cultivar em estudo, local e a época da colheita, tendendo a aumentar com o amadurecimento dos frutos devido a diminuição da acidez.

\section{Conclusão}

Entre os tratamentos analisados no presente trabalho, a melhor forma de armazenamento póscolheita de frutos de $P$. tenuifila é a condição refrigerada com temperatura de $10{ }^{\circ} \mathrm{C}$ e $90 \%$ de umidade relativa.

Frutos de $P$. tenuifila produzidos em sistema convencional e orgânico possuem a mesma vida útil após a colheita. A acidez titulável não varia durante o armazenamento pós-colheita desses frutos.

A embalagem de PEAD com $30 \mu \mathrm{m}$ de espessura é recomendada como auxiliar na manutenção da vida útil dos frutos de $P$. tenuifila reduzindo a perda de massa fresca, manutenção da textura, cor e características físico-químicas.

\section{Agradecimentos}

Os autores agradecem ao Conselho Nacional de Desenvolvimento Científico e Tecnológico (CNPq) projeto $n^{\circ} 404847 / 2012-09$ pelo apoio financeiro, bolsas de Iniciação Científica (PIBIC/CNPQ) e à Empresa Brasileira de Pesquisa Agropecuária (Embrapa) pelo apoio no desenvolvimento do projeto de pesquisa. 


\section{Literatura Citada}

\section{AMERICAN MEAT SCIENCE ASSOCIATION} MEAT - AMSAM. 2012. Meat color measurement guidelines. Savoy: American Meat Science Association.

ANVISA - Agência Nacional de Vigilância Sanitária. Legislação. Brasília, 2018. Disponível em: https:/ /www.in.gov.br/materia/-/asset_publisher/ Kujrw0TZC2Mb/content/id/54522298/do1-201812-11-resolucao-de-diretoria-colegiada-rdc-n-255de-10-de-dezembro-de-2018-54521871 Acesso em: 10 jan. 2021.

ASSOCIATION OF OFFICIAL ANALYTICAL AOAC. 2016. Official methods of analysis of the Association of Official Analytical Chemists (20th ed.) Gaithersburg: AOAC.

BOTELHO, S. C. C. et al. 2019. Qualidade póscolheita de frutos de maracujazeiro-amarelo colhidos em diferentes estádios de maturação. Revista Ciências Agrárias 62:1-8.

CARLOSAMA, A. R. et al. 2020. Pasifloras: especies cultivadas en el mundo. Brasília, DF: ProImpress. 249p. il.

D'ABADIA, A. C. A. et al. 2020. Determination of the maturation stage and characteristics of the fruits of two populations of Passiflora cincinnata Mast. Revista Caatinga 33(2):349-360.

FALEIRO, F. G. et al. 2017. Espécies de maracujazeiro no mercado internacional. In: Junghans, T. G.; Jesus, O. N. Maracujá: do cultivo à comercialização. Brasília, DF, Embrapa. pp.15-37.

FALEIRO, F. G. et al. 2018. Cultivar de maracujazeiro medicinal da espécie Passiflora tenuifila Killip como alternativa para o mercado de frutas especiais com propósitos de processamento de produtos funcionais medicinais BRS VF (BRS Vita Fruit). Embrapa. Folder. Disponível em: https://ainfo.cnptia.embrapa.br/digital/bitstream/ item/197024/1/BRS-Vita-fruit-web.pdf

FALEIRO, F. G. et al. 2020. O gênero Passiflora: diversidade, conservação e uso. In: Faleiro, F. G.; Oliveira, J. S.; Walter, B. M. T.; Junqueira, N. T. V. Banco de germoplasma de Passiflora L. 'Flor da Paixão': caracterização fenotípica, diversidade genética, fotodocumentação e herborização. Brasília, DF: ProImpress. p. 9-16.

FEDERAÇÃO DA AGRICULTURA DO ESTADO DO PARANÁ - FAEP. Classificação do maracujá-amarelo. 2008. Disponível em: http:// www.faep.com.br/comissoes/frutas/cartilhas/ frutas/maracuja.htm. Acesso em: 09 jun. 2021.

FERREIRA, D. F. 2019. Sisvar: a computer analysis system to fixed effects split plot type designs. Revista Brasileira de Biometria 37(4):529-535.

FLOROS, J. D.; MATSOS, K. I. 2005. Introduction on modified atmosphere packaging. In: Han, J. $\mathrm{H}$. Innovations in food packaging. Disponível em: $<$ http://books.google.com.br. Acesso em: 20 ago. 2021.

HOLANDA, D. K. R. et al. 2020. Garlic passion fruit (Passiflora tenuifila Killip): Assessment of eventual acute toxicity, anxiolytic, sedative, and anticonvulsant effects using in vivo assays. Food Research International 128:108813.

HOLANDA, D. K. R. et al. 2019. Passiflora tenuifila Killip: Assessment of chemical composition by ${ }^{1} \mathrm{H}$ NMR and UPLC-ESI-Q-TOF-MS ${ }^{\mathrm{E}}$ and its bioactive properties in a rotenone-induced rat model of Parkinson's disease. Journal of Functional Foods 62:103529.

HOLANDA, D. K. R. 2015. Desenvolvimento e caracterização de sopa de vegetais e carne a base de maracujá silvestre (Passiflora tenuifila Killip) submetida ao processo de esterilização. Dissertação Mestrado, Florianópolis, UFC. 71p.

HUNTERLAB, 1996. Insight on color: Hunter LabColor Scale. Applications Note. 8(9):1-15.

KADER, A. A. 2010. Future of Modified Atmosphere Research. Acta Horticulturae 857:212-217.

LIMA, L. A. S. et al. 2014. Caracterização dos solos da Bacia do Sarandi. Planaltina, DF, Embrapa Cerrados, Boletim de Pesquisa e Desenvolvimento, v.318, 47p.

MOURA, G. S. et al. 2016. Conservação pós-colheita de frutos de maracujá-amarelo por derivados de capim-limão (Cymbopogon citratus). Ambiência 12(2):667-682. 
PEREIRA, R. C. A.; SILVEIRA, M. R. S.; COSTA, A. M. 2017. Maracujá silvestre (Passiflora tenuifila Killip) aspectos agronômicos e característicos dos frutos. Fortaleza, CE: Embrapa Agroindústria Tropical. 5p.

PEREIRA, L. D. et al. 2018. Caracterização de frutos de diferentes espécies de maracujazeiro. Revista Brasileira de Agropecuária Sustentável 8(2):21-28.

RAMOS, A. R. P. et al. 2013. Qualidade de frutos de tomate 'giuliana' tratados com produtos de efeitos fisiológicos. Semina: Ciências Agrárias 34(6):3543-3552.

RINALDI, M. M.; DIANESE, A. C.; COSTA, A. M. 2021. Avaliação do uso de cera de carnaúba na conservação pós-colheita de frutos de Passiflora cincinnata cv. BRS Sertão Forte. Agrotrópica 33(1):75-84.

RINALDI, M. M. et a1. 2019a. Post-harvest conservation of Passiflora alata fruits under ambient and refrigerated condition. Ciência e Tecnologia de Alimentos 1:1-8.

RINALDI, M. M. et al. 2019b. Atmosfera modificada na conservação pós-colheita de frutos de Passiflora alata cv. BRS Mel do Cerrado (BRS MC). Agrotrópica 31(3):185-196.

RINALDI, M. M. et al. 2017a. Conservação póscolheita de frutos de Passiflora setacea DC. submetidos a diferentes sanitizantes e temperaturas de armazenamento. Brazilian Journal of Food Technology 20:1-12e2016046.
RINALDI, M. M. et al. 2017b. Recomendações de manuseio e conservação pós-colheita de frutos de Passiflora setacea e Passiflora alata. Brasília - DF: Embrapa, Comunicado Técnico. 4p.

RINALDI, M. M. et al. 2009. Estabilidade de repolho minimamente processado sob diferentes sistemas de embalagem. Food Science and Technology,29(2):310-315.

SILVA, A. C. G.; SILVA, N. S.; SOUSA, F. F. 2019. Pós-colheita do maracujá amarelo com revestimentos a base de amido da entrecasca de mandioca. Revista Verde 14(2):238-245.

SILVEIRA, M. R. S. et al. 2019. Composição físicoquímica e bioativa dos frutos de Passiflora tenuifila Killip (maracujá-alho). Revista Cubana de Plantas Medicinales 24(1):75-81.

SOLINO, A. J. S. et a1. 2012. Severidade da antracnose e qualidade dos frutos de maracujáamarelo tratados com produtos naturais em póscolheita. Revista Brasileira de Fruticultura 34(1):57-66.

TAVARES, J. T. Q. et al. 2003. Aplicação pós-colheita de cloreto de cálcio em maracujá amarelo. Magistra 15(1):7-12.

ZACHARIAS, A. O.; FALEIRO, F. G.; ALMEIDA, G. Q. 2020. Producers profile and the adoption of technologies in passion fruit cultivation in the Triângulo Mineiro region. Revista Brasileira de Fruticultura 42(5):e-058. 